\title{
IDENTIFICACIÓN MOLECULAR DE CLONES Salix MEDIANTE RAPDS ${ }^{(*)}$
}

\section{HUGO CAMPOS DE QUIROZ (**)}

(*) Investigación financiada mediante los proyectos FDI PF-05 y FONDEF D96I1074

(**) INIA-CRI Carillanca, Casilla 58-D, Temuco, Chile. E-mail: hcampos@carillanca.inia.cl

\section{RESUMEN}

En la actualidad, un importante esfuerzo de investigación desarrolla el cultivo del mimbre y otras especies Salix como una alternativa de diversificación forestal en Chile. Debido a su propagación vegetativa, los mecanismos de identificación genética juegan un rol importante en el desarrollo de estas especies en Chile. Los sistemas tradicionales de identificación de especies y genotipos forestales, se basan principalmente en características morfologicas y fenologicas, cuya expresión presenta una gran dependencia del medio ambiente y del conocimiento de la especie. En consecuencia, es necesario desarrollar sistemas que permitan la rápida y precisa identificación de genotipos forestales. El desarrollo de marcadores moleculares RAPDs (Random Amplified Polymorphic DNA) permite una rápida identificación de clones y otros genotipos forestales mediante la amplificación selectiva de fragmentos del genoma de los individuos analizados. El presente estudio demuestra la utilidad de este enfoque, mediante la identificación molecular de un diverso grupo de procedencias Salix. A través de RAPDs, y utilizando un solo partidor, fue posible identificar un conjunto de 24 procedencias analizadas. Los resultados obtenidos se discuten en términos de sus consecuencias genéticas y operativas, tanto para productores y viveristas como, para el desarrollo silvícola de especies Salix en Chile.

Palabras Clave: Salix, RAPDs, Biotecnología, Genética Forestal

\section{SUMMARY}

An important research effort is currently underway in Chile in order to develop basket willows and other Salix species as an alternative for forest diversification. Due to their vegetative reproduction, genetic identification systems are required for this group of species. Traditional systems of forest species and genotypes identification are based upon morpho-and phenological characteristics which are influenced to a significant extent by enviromental conditions and the knowledge available for a given species. Therefore, systems enabling the steady and accurate identification of forest genotypes need to be developed.The development of a system of molecular markers called RAPDs (Random Amplified Polymorphic DNA) allows the quick identification of clones and other forest genotypes through the selective amplification of genome fragments from the analyzed individuals. The usefulness of this approach is shown in this study, which aimed to identify with RAPDs a group of Salix provenances. Using a single primer twenty four provenances were identified. Results are discussed in terms of their genetic, productive and operative consequences

Keywords: Salix, RAPDs, Biotechnology, Forest Genetics 


\section{INTRODUCCIÓN}

La búsqueda de nuevas alternativas forestales rentables para los pequeños agricultores en Chile, se hace necesaria al considerar que aún aquellas alternativas forestales tradicionales de ciclo corto, como eucalipto y álamo, no representan una solución para el campesinado debido a que implican períodos de espera en la obtención de beneficios superiores a los 8 años. En consecuencia, es necesario desarrollar y adaptar tanto nuevas alternativas forestales de ciclo corto como la tecnología de manejo asociada. Uno de los grupos más promisorios de diversificación forestal para nuestro país corresponde al género Salix.

Las especies e híbridos interespecíficos del género Salix (aproximadamente 300) comparten una serie de propiedades como rápido crecimiento, eficiente uso de agua y nutrientes, fácil regeneración, tolerancia a la competencia y buena adaptación al estrés, representando, por lo tanto, una atractiva alternativa de diversificación para el sector forestal chileno. A nivel mundial, bajo condiciones de silvicultura intensiva, estas especies sustentan la producción de energía, compuestos químicos y fibras en naciones como Canadá, Suecia e Inglaterra (Zsuffa, 1984). En nuestro país, ciertas especies como $S$. viminalis (mimbre) sustentan una importante industria artesanal de producción de muebles, la cual mediante su tecnificación presenta un buen potencial de exportación y competencia con alternativas como el ratán, mientras que otras especies Salix pueden ser utilizadas con fines como la estabilización de cuencas. La distribución geográfica del mimbre en Chile comprende las regiones IV a XI, concentrándose principalmente en la VI región, en la zona de Chimbarongo.

A diferencia de otras especies forestales, el material comúnmente utilizado para establecer plantaciones de Salix corresponde a clones desarrollados mediante propagación vegetativa a partir de individuos seleccionados. Así mismo, la hibridación interespecífica es un fenómeno frecuente dentro de este género. En consecuencia, el mejoramiento genético de la especie presenta el potencial no sólo de capturar heterosis mediante el desarrollo de clones híbridos, sino también de capturar componentes no aditivos de la varianza genética, los cuales no son transmitidos mediante reproducción sexual.

La descripción tradicional de especies y genotipos Salix, se basa en numerosos caracteres morfológicos como sexo, tipo de copa, color y pilosidad del fuste, número y distribución de lenticelas, ángulo de ramificación, características del follaje, persistencia estipular y otras (Stott, 1988), las cuales a la vez de exigir un adecuado conocimiento botánico de las especies, dependen del medio ambiente para su expresión y deben ser evaluadas dentro de precisos lapsos de tiempo, lo cual limita su aplicación operativa masiva. Con el objeto de monitorear la eficiencia operacional de viveristas, productores, y en general de los agentes de la cadena de comercialización basada en Salix, se hace necesario desarrollar sistemas objetivos de identificación genotípica, que permitan 
establecer con un alto grado de certeza el origen genético de un clon determinado. Una alternativa tecnológica al respecto es el uso de isozimas, las cuales si bien permiten resolver ciertas interrogantes de orden filogenético, por lo general carecen del poder resolutivo necesario para identificar individuos forestales (Khasa y Dancik, 1996; Van de Ven y McNicol, 1995).

El reciente desarrollo de marcadores moleculares (Campos de Q., 1995) ofrece una nueva alternativa analítica tanto para especies Salix como para cualquier otro género de importancia forestal. Los marcadores moleculares permiten identificar variabilidad genética directamente al nivel de ADN (Ácido Desoxirribonucleico), y por lo tanto, poseen un poder resolutivo mucho mayor que otras alternativas como proteínas de reserva, isozimas o metabolitos secundarios como terpenos. Además, ellos carecen de efectos ambientales, por lo tanto su información es estable a través del tiempo y la ontogenia de un individuo.

Dentro de los marcadores moleculares, aquellos que presentan un mayor potencial de aplicación forestal corresponden a los basados en procesos PCR (Polymerase Chain Reaction), los cuales amplifican selectivamente fragmentos de ADN de un individuo analizado. Tal es el caso de los RAPDs (Random Amplified Polymorphic DNA) (Williams et al., 1990), los cuales presentan las siguientes ventajas:

1) Requieren ínfimas cantidades de $\mathrm{ADN}$.

2) No requieren radioactividad.

3) Permiten generar información con mayor velocidad que otras alternativas tecnológicas

4) Han demostrado su versatilidad y utilidad en numerosas especies forestales.

Una completa descripción de la Polymerase Chain Reaction y las bases genéticas y moleculares de los RAPDs se encuentran en Campos de Q. (1995) y Campos de Q. y Salvo (1995). RAPDs se comparan ventajosamente con otros marcadores moleculares como RFLPs e isozimas en especies forestales (Liu y Furnier, 1993). Entre las aplicaciones de los marcadores moleculares en el área forestal destacan la construcción de mapas genéticos, la identificación de poligenes regulando caracteres de importancia económica, el análisis de identidad clonal y el determinar relaciones genéticas en numerosas especies forestales como Eucalyptus, Picea y Populus, entre numerosas otras (Gratapaglia y Sederoff; Van der Ver y McNicol, 1995 y Castiglione et al., 1993).

El presente trabajo muestra una de las primeras aplicaciones de marcadores moleculares en el área forestal en nuestro país, el desarrollo de un protocolo RAPDs para varias especies e híbridos Salix y su utilización para la identificación clonal en estas especies. 


\section{OBJETIVOS}

Los objetivos del presente trabajo son:

1) Desarrollar un protocolo RAPDs para especies Salix

2) Identificar molecularmente clones Salix mediante RAPDs

\section{MATERIAL Y MÉTODOS}

El material vegetal empleado correspondió a plantas in vitro de las especies y procedencias indicadas en el Cuadro 1.

Cuadro 1.

ESPECIES Y PROCEDENCIAS Salix ANALIZADAS

\begin{tabular}{|c|l|l|}
\hline Código & Especie & Procedencia \\
\hline a & Salix viminalis & UK Bowles Hybrid \\
\hline b & Salix viminalis & UK Mulatin \\
\hline c & Salix viminalis & UK 455 De Biardii \\
\hline d & Salix viminalis & SWE 78183 \\
\hline e & Salix viminalis & SWE 79046 \\
\hline f & Salix viminalis & SWE 78112 \\
\hline g & Salix purpurea & CAN Ontario Pur 12 \\
\hline h & Belgicum Red & Holanda \\
\hline i & Salix dasyclados & UK \\
\hline j & Salix dasyclados & Swe 77056 \\
\hline k & Salix dasyclados & Swe 79097 \\
\hline l & Salix dasyclados & Swe 81090 \\
\hline m & Salix burjatica & UK Korso \\
\hline n & Salix burjatica & UK Germany \\
\hline o & Salix burjatica & UK-83 \\
\hline p & Salix calodendron & UK \\
\hline q & Salix stipularis & UK \\
\hline r & Salix sericans & UK Coles \\
\hline s & Salix sericans & UK Reifenweide \\
\hline t & Salix eriocephala & Can Ontario 558 \\
\hline u & Salix eriocephala & Can Ontario ER63 \\
\hline v & Salix eriocephala X pentandra & Can Ontario 601 \\
\hline w & Salix eriocephala X exigua & Can Ontario 611 \\
\hline x & China shrub & Can Ontario S-67 \\
\hline & & \\
\hline
\end{tabular}

\section{Extracción de ADN}

La extracción de ADN a partir de procedencias Salix se dificulta debido a la presencia de grandes cantidades de fenólicos, carbohidratos y otros compuestos. Partiendo del protocolo base de Ojeda et al. (1998), se desarrolló un protocolo que 
permite obtener $\mathrm{ADN}$ en adecuada cantidad y apropiada calidad, utilizando un tampón de extracción basado en el detergente CTAB, y posterior separación de fases mediante cloroformo. El ADN se cuantificó mediante lecturas espectrofotométricas a $\lambda 260$ o fluorométricas a $\lambda 465$, y la calidad del $\mathrm{ADN}$ se determinó mediante el análisis espectrofotométrico $\lambda 260 / 280$. La integridad general del ADN se verificó mediante una corrida electroforética en gel de agarosa. Una vez diluido, el ADN fue disuelto en buffer $1 \mathrm{XTE}$ y mantenido en tubos Eppendorf a $-20^{\circ} \mathrm{C}$. Todos los reactivos utilizados para extraer ADN, enzimas, reactivos PCR, así como los compuestos empleados en las etapas posteriores del análisis, correspondieron a reactivos del tipo pureza biología molecular.

\section{Desarrollo de RAPDs}

Se desarrolló un protocolo que permite la rápida y eficaz obtención de RAPDs en Salix spp. A objeto de desarrollar de modo eficiente un protocolo RAPDs para estas especies, se determinaron las condiciones óptimas PCR (Polymerase Chain Reaction) necesarias. Los partidores utilizados fueron adquiridos de Operon Technologies (USA), y corresponden a decámeros con un contenido de Citosina +Guanina entre 60 y $70 \%$. La totalidad de las reacciones anteriores fueron cubiertas con aceite mineral y desarrolladas en un termociclador Perkin Elmer 480.

\section{Separación de Productos RAPDs}

Los productos RAPDs fueron separados mediante electroforesis horizontal en geles de agarosa (Gibco BRL). El tampón utilizado para los geles y el tampón de corrida fue $1 \mathrm{X}$ TAE. La concentración de agarosa fluctuó entre 1 y $2 \%$ en función del tamaño de los productos PCR a separar. Como marcador de peso molecular se utilizó $1 \mathrm{~kb}$ ladder (Promega). Una vez finalizado el análisis electroforético, los geles fueron teñidos con una solución $1 \mu \mathrm{g} / \mathrm{ml}$ de Bromuro de Etidio y posteriormente visualizados en un transiluminador UV. Las imágenes de los geles de agarosa se procesaron mediante un sistema digital Kodak KD1D, siendo grabadas y posteriormente procesadas.

\section{RESULTADOS Y DISCUSIÓN}

\section{Extracción de ADN}

El protocolo original utilizado generó ADN de calidad insuficiente en términos de degradación de $\mathrm{ADN}$, por lo tanto se evaluaron varias modificaciones. Los mejores resultados se obtuvieron al agregar compuestos antioxidantes y protectores como 2- $\beta$ Mercaptoetanol y PVPP al buffer de extracción. Particularmente, la adición de PVPP al tampón de extracción permitió incrementar la cantidad de ADN de alto peso molecular extraído y reducir al mínimo su degradación. En el Cuadro 2 se presenta la concentración de ADN extraida por genotipo. 
Las concentraciones de $\mathrm{ADN}$ obtenidas fluctuaron entre $1.250 \mathrm{ng} / \mu \mathrm{l}$ (procedencia $S$. eriocephala Can Ontario 558) y $315 \mathrm{ng} / \mu \mathrm{l}$ (procedencia $S$. burjatica UK Korso) (Cuadro 2). Tales variaciones se encuentran dentro del rango normal observado en especies vegetales.

\section{Cuadro 2.}

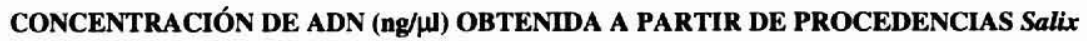

\begin{tabular}{|c|c|c|c|c|c|}
\hline $\begin{array}{c}\text { Código } \\
\text { Procedencia }\end{array}$ & $\begin{array}{c}\text { Conc. de ADN } \\
(\mathrm{ng} / \mu \mathrm{l})\end{array}$ & $\begin{array}{c}\text { Código } \\
\text { Procedencia }\end{array}$ & $\begin{array}{c}\text { Conc. de ADN } \\
(\mathrm{ng} / \mu \mathrm{l})\end{array}$ & $\begin{array}{c}\text { Código } \\
\text { Procedencia }\end{array}$ & $\begin{array}{c}\text { Conc. de ADN } \\
(\mathrm{ng} / \mu \mathrm{l})\end{array}$ \\
\hline $\mathrm{a}$ & 970 & $\mathrm{i}$ & 380 & $\mathrm{q}$ & 845 \\
\hline $\mathrm{b}$ & 585 & $\mathrm{j}$ & 805 & $\mathrm{r}$ & 500 \\
\hline $\mathrm{c}$ & 530 & $\mathrm{k}$ & 1.125 & $\mathrm{~s}$ & 565 \\
\hline $\mathrm{d}$ & 385 & $\mathrm{l}$ & 905 & $\mathrm{t}$ & 410 \\
\hline $\mathrm{e}$ & 1.250 & $\mathrm{~m}$ & 1.035 & $\mathrm{u}$ & 435 \\
\hline $\mathrm{f}$ & 450 & $\mathrm{n}$ & 385 & $\mathrm{v}$ & 305 \\
\hline $\mathrm{g}$ & 350 & $\mathrm{o}$ & 420 & $\mathrm{w}$ & 470 \\
\hline $\mathrm{h}$ & 340 & $\mathrm{p}$ & 315 & $\mathrm{x}$ & 710 \\
\hline
\end{tabular}

En cuanto a la relación espectrofotométrica $\lambda 260 / 280$, la cual indica la calidad y pureza del ADN extraído, los valores observados fluctuaron entre 1,45 y 2,2, rango considerado como adecuado. Al comparar la optimización del sistema de extracción de ADN para especies como trébol rosado, trigo y cebada, y la requerida para procedencias Salix, este último grupo de especies necesitó una mayor cantidad de modificaciones y precauciones. Sin embargo, finalmente generó ADN de adecuada calidad para sustentar reacciones PCR. El protocolo desarrollado para Salix permite procesar un buen número de muestras por día, y además no genera desechos tóxicos de difícil eliminación como fenol. Del mismo modo, evita la utilización de compuestos de elevado costo como Cloruro de Cesio, el cual ha sido utilizado para purificar muestras de ADN producidas a partir de especies forestales.

\section{Optimización de la Reacción RAPDs}

Una reacción RAPDs implica numerosos componentes que interactúan entre sí de modo complejo. En consecuencia, se hace necesario optimizar sus concentraciones. Utilizando la metodología indicada en Ojeda et al. (1998), se determinaron las condiciones RAPDs óptimas para Salix, las cuales corresponden a $0,5 \mathrm{mM}$ de $\mathrm{Mg}^{+2} ; 0,2$ $\mathrm{mM}$ dNTPs (Promega); 25 pmoles de partidores (Operon Technologies), 1 unidad de Taq polimerasa (Gibco BRL), 2,5 $\mu$ l de buffer PCR (Gibco BRL) y $50 \mathrm{ng}$ de ADN en un volumen final de $25 \mu \mathrm{l}$.

Los partidores utilizados correspondieron a secuencias artificiales de ADN de 10 nucleótidos de extensión con un contenido de Citosina + Guanina entre 60 y $70 \%$. Un total de 42 partidores fueron evaluados en genotipos Salix seleccionados, a objeto de 
identificar aquellos que permitan detectar la mayor cantidad de variabilidad genómica en esta especie. De este modo se aceleró el desarrollo del protocolo RAPDs necesario para esta especie forestal. En el Cuadro 3, se presentan los partidores Operon evaluados y aquellos seleccionados para su utilización amplia en las procedencias estudiadas.

Cuadro 3.

PARTIDORES OPERON EVALUADOS

\begin{tabular}{|c|c|c|c|c|c|}
\hline Partidor & Resultado & Partidor & Resultado & Partidor & Resultado \\
\hline AK01 & X & BA06 & $\checkmark$ & AB03 & X \\
\hline AK04 & $\checkmark$ & BA07 & $\checkmark$ & AB04 & X \\
\hline AK07 & X & BA08 & $\checkmark$ & AB10 & X \\
\hline AL07 & X & BA10 & $\checkmark$ & AB14 & X \\
\hline AL09 & X & BA14 & $\checkmark$ & AB16 & X \\
\hline AL15 & X & AA06 & X & AB18 & X \\
\hline AC06 & X & AA07 & $\checkmark$ & AB20 & X \\
\hline AC10 & X & AA09 & X & AC06 & X \\
\hline AC12 & X & AA10 & $\checkmark$ & AC10 & X \\
\hline AC17 & X & AA13 & X & AC12 & X \\
\hline AC20 & X & AA14 & $\checkmark$ & AC13 & X \\
\hline AV20 & X & AA17 & $\checkmark$ & AC14 & X \\
\hline BA01 & $\checkmark$ & AA18 & X & AC16 & X \\
\hline BA02 & X & AA20 & X & AC17 & X \\
\hline
\end{tabular}

$\checkmark$ : Resultado positivo

Del total de 42 partidores evaluados, $10(23,8 \%)$ de ellos generaron RAPDs de calidad apropiada. Este valor porcentual es similar a los alcanzados con otras especies forestales (Furman et al., 1997). Numerosos partidores resultaron útiles en Salix, todos los cuales son completamente distintos al nivel de secuencia de ADN y consecuentemente, amplifican fragmentos de $\mathrm{ADN}$ de diferente naturaleza. Lo anterior permite analizar distintas regiones del genoma Salix y por lo tanto generar información de mayor confiabilidad.

En relación al ciclo térmico utilizado en un termociclador Perkin Elmer 480, este consistió de las siguientes etapas: $1 \mathrm{~min}$. a $94^{\circ} \mathrm{C}$ y posteriormente 42 ciclos de $0,5 \mathrm{~min}$. a $94^{\circ} \mathrm{C}, 1 \mathrm{~min}$. a $35^{\circ} \mathrm{C}$ y $1,5 \mathrm{~min}$. a $72^{\circ} \mathrm{C}$. Como testigos negativos se utilizaron reacciones incluyendo agua estéril en lugar de templado.

\section{Reproducibilidad de la Reacción RAPDs}

Frecuentemente se indica que una de las principales limitantes del uso de RAPDs es su reproducibilidad, debido a que la naturaleza molecular del sistema es distinta a la de otros marcadores moleculares como RFLPs (Restriction Fragment Length Polymorphisms). A objeto de evaluar la reproducibilidad del proceso, extracciones independientes de ADN del mismo genotipo fueron sometidas a procesos RAPDs 
totalmente independientes. La Figura 1 indica los resultados obtenidos a partir de reacciones RAPDs independientes entre sí, los cuales muestran que la reproducibilidad obtenida es adecuada. La reproducibilidad de los RAPDs logrados en el presente estudio con procedencias Salix fue similar a los valores obtenidos para otras especies vegetales en nuestro país (Ojeda et al., 1998). Un estricto control de las condiciones experimentales permite alcanzar tales valores de reproducibilidad, lo que concuerda con Teasdale (1996), quien indica valores de reproducibilidad de RAPDs en especies forestales superiores a $95 \%$. De forma rutinaria durante el estudio se incluyeron muestras duplicadas, obteniéndose en todos los casos idénticos resultados a partir de ellas.

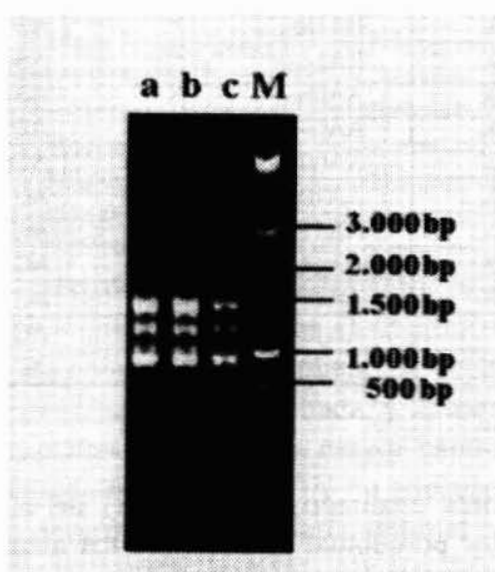

Figura 1. REPRODUCIBILIDAD DE RAPDs EN Salix spp. MUESTRAS 1 A 4 CORRESPONDEN AL RESULTADO DE 4 AMPLIFICACIONES INDEPENDIENTES. EL TAMAÑO DE LAS BANDAS SE EXPRESA EN PARES DE BASES. M: MARCADOR DE PESO MOLECULAR.

\section{Análisis de la Información RAPDs Generada}

El resultado de la amplificación PCR obtenida mediante RAPDs corresponde a un conjunto de fragmentos independientes de ADN, los cuales se separan entre sí principalmente en función de su peso molecular, mediante electroforesis en geles de agarosa. Los fragmentos RAPDs obtenidos generalmente fluctuaron entre 550 y $3.500 \mathrm{pb}$ (pares de bases), rango similar al observado en otras especies forestales analizadas mediante este tipo de marcador molecular.

Una vez identificados 10 partidores Operon que generaron información adecuada, 24 procedencias Salix fueron sometidas a un análisis completo con los partidores indicados. 
Los valores de polimorfismo encontrados son elevados, lo cual confirma la gran variabilidad existente dentro del género Salix. El porcentaje de RAPDs polimórficos fue adecuado y similar al determinado para Nicotiana (95,7\%) (Yu y Lin, 1997). El número de polimorfismos generados por partidor alcanzó un valor promedio de 8,5 , el cual se compara favorablemente con los valores obtenidos en otras especies forestales, puesto que en Picea spp., el número de polimorfismos por partidor reportado fluctúa entre 3,8 y 5,2 (Mosseler et al., 1992), y alcanza a 9,7 en Picea abies (Bucci y Menozzi, 1993). De este modo, RAPDs constituyen un apropiado marcador molecular para el análisis de especies Salix y otras de importancia forestal, debido a que una elevada proporción de ellos son polimórficos y en consecuencia generan información de utilidad. Además, es importante considerar que la velocidad analítica que ellos permiten es sustantivamente superior a la obtenida mediante otros marcadores moleculares, parámetro de importancia al considerar la incorporación de marcadores moleculares al análisis de especies forestales en Chile.

\section{Diferenciación Molecular de Procedencias Salix}

La premisa fundamental de la identificación de clones forestales mediante RAPDs es que la diferencia genética existente entre ellos debe reflejarse consistentemente en la información molecular recopilada mediante RAPDs. En consecuencia, si dos procedencias Salix son genéticamente distintas entre sí, tal diferencia debiera ser reflejada en la información generada mediante RAPDs. La Figura 2 presenta el resultado del análisis RAPDs efectuado con el partidor AK04. Se observa que mediante RAPDs es posible obtener una gran cantidad de fragmentos de ADN variables entre distintas procedencias. Además, ellos se presentan dentro de un amplio rango de peso molecular, lo cual permite analizar distintas áreas genómicas, incrementando así la confiabilidad del análisis.

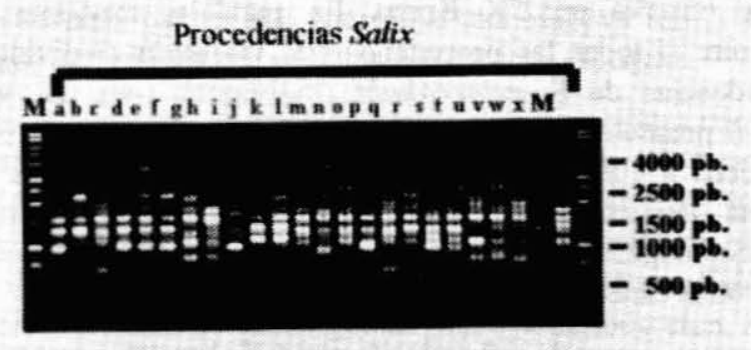

Figura 2. RAPDs OBTENIDOS CON EL PARTIDOR AK04 EN LAS 24 PROCEDENCIAS Salix spp. ANALIZADAS. EL TAMAÑO DE LAS BANDAS SE EXPRESA EN PARES DE BASES. M: MARCADOR DE PESO MOLECULAR. 
A nivel molecular existe una gran cantidad de variabilidad genética, tanto entre especies como entre procedencias de la misma especie Salix, con todas las procedencias analizadas presentando un patrón RAPDs distinto no sólo para el partidor AK04 sino también para otros partidores evaluados.

Una aplicación directa e inmediata del protocolo RAPDs desarrollado es generar sistemas de identificación molecular de clones Salix. En la Figura 2 se observa que aquellas procedencias de la misma especie presentan numerosas bandas comunes, y además la presencia de bandas distintas que permiten diferenciarlas entre ellas (Figura 2). De este modo, es factible asumir que clones Salix con un patrón RAPDs similar poseen un origen genético común, mientras que aquellos que presentan patrones RAPDs disímiles provienen de orígenes genéticos diversos. Ejemplo de esto lo constituye la procedencia China shrub Can Ontario S-67, la cual no corresponde a ninguna de las otras especies Salix incluidas en el estudio, puesto que en la Figura 2 se observa que los fragmentos de ADN amplificados a partir de ellas, es distinto al observado para todos los otros genotipos Salix incluidos en el presente análisis. En consecuencia, la información RAPDs refleja fielmente diferencias de origen genético entre las procedencias estudiadas.

El análisis RAPDs desarrollado no sólo permite diferenciar procedencias Salix pertenecientes a distintas especies, del mismo modo posibilita la efectiva diferenciación de procedencias de la misma especie. Al respecto, y como debiera esperarse, todas las procedencias $\boldsymbol{S}$. dasyclados presentan un patrón RAPDs muy similar, no obstante lo cual fue posible diferenciarlas a todas ellas. Además, fue posible la diferenciación molecular de las procedencias Swe 79097 y Swe 81090, las cuales poseen un origen genético similar (Suecia), mediante la presencia de dos fragmentos RAPDs: uno de aproximadamente 1.100 pares de bases exclusivamente presentes en Swe 79097, y un segundo fragmento de tamaño levemente inferior a 1.500 pares de bases, inexistente en Swe 81090 . Del mismo modo, las procedencias Swe 81090 y UK Korso se diferencian entre sí mediante un fragmento de aproximadamente 900 pares de bases, el cual solamente se observa en UK Korso. En paralelo, mediante RAPDs fue posible diferenciar entre sí todas las procedencias $\boldsymbol{S}$. viminalis (individuos a-f) así como las cuatro procedencias de $\mathbf{S}$. eriocephala (individuos $\mathrm{t}-\mathrm{w}$ ). La valiosa diferenciación obtenida en el presente estudio, aún entre procedencias de la misma especie mediante RAPDs, se debe a que este sistema de marcadores moleculares permite un grado de diferenciación molecular sustantivamente superior al obtenido mediante otras alternativas tecnológicas, lo cual concuerda con los análisis moleculares intraespecíficos obtenidos en otras especies vegetales (dos Santos et al., 1994).

La información generada refleja la efectividad obtenida a través del uso de RAPDs en procedencias Salix, puesto que mediante el uso de solamente 1 partidor fue posible inequívocamente discriminar entre las procedencias analizadas. En contraposición, en álamo fue necesario utilizar 12 tinciones isozímicas para identificar un grupo de 27 clones (Comisión Nacional del Alamo, 1997). En Picea sitchensis el número mínimo de 
partidores requeridos para diferenciar un grupo de genotipos fue 4 (Van de Ven y McNicol, 1995), lo cual indica tanto la gran variabilidad genética existente dentro del género Salix, como el potencial que presenta para la utilización de marcadores moleculares.

Existe un reducido número de especies forestales en las cuales RAPDs han sido utilizados con propósitos de identificación genotípica y/o clonal. En Picea abies, por ejemplo, mediante RAPDs se generó un catálogo molecular de clones seleccionados (Scheepers et al., 1994), mientras que en Picea glauca y $\boldsymbol{P}$. engelmanii e híbridos naturales, RAPDs permitieron generar protocolos de rápida identificación clonal (Khasa y Dancik, 1996). Por su parte, en $\boldsymbol{P}$. Sitchensis, RAPDs permitieron la identificación de 57 árboles plus pertenecientes a un programa de mejoramiento genético, lo que facilita su manejo operativo (Van de Ven y McNicol, 1995). Los resultados obtenidos en este estudio posibilitan el desarrollo de sistemas moleculares de identificación clonal para clones y/o especies Salix de importancia para nuestro país.

\section{Integración de la Tecnología RAPDs al Desarrollo Silvícola y Manejo de Procedencias Salix en Chile}

$\mathrm{La}$ información generada indica que RAPDs podrían ser utilizados en sistemas de certificación molecular de identidad genética, permitiendo así proteger la propiedad intelectual derivada de procedencias identificadas por su adecuado comportamiento en Chile y/o mediante el mejoramiento genético de la especie en nuestro país. Puesto que la información RAPDs es estable en el tiempo, una alternativa es utilizarla para conformar una base de datos en la cual cada clon Salix se represente por un patrón de fragmentos de ADN específico, de modo análogo al sistema de "DNA fingerprints" (huella dactilar del ADN) usado en aplicaciones forenses, lo cual presenta el potencial de ser automatizado e informatizado para generar así un sistema análogo a los códigos de barra utilizados.

Lo anterior representaría un importante estímulo a la investigación genética con especies Salix en nuestro país, puesto que constituye una efectiva forma para proteger propiedades intelectuales derivadas y sus ventajas económicas asociadas.

En paralelo, y considerando que el establecimiento de plantaciones comerciales con genotipos Salix se basa en material propagado vegetativamente, el desarrollo de un sistema que permita identificar con gran certeza y rapidez la constitución genética de un clon dado, es de gran importancia operacional, puesto que permitiría monitorear la eficiencia operacional de los viveros productores de genotipos y clones Salix en nuestro país mediante el análisis molecular de la integridad genética de todo clon, reduciéndose así riesgos de clasificaciones y comercialización errada de clones Salix.

Estudios de identificación clonal en otras especies forestales han determinado significativas e inesperadas tasas de error. En $\boldsymbol{P}$. abies, el análisis de seis genotipos elite y sus respectivos rametos derivados, indicó en uno ( $16 \%$ del total) de ellos la presencia 
de rametos mal identificados (Scheepers et al., 1997). En P. Taeda, mediante isozimas han sido identificadas tasas de errores de clasificación de clones y rametos fluctuantes entre 2 y 13\% (Wheeler y Jech, 1992). Considerando que estos últimos valores se basan en isozimas, las cuales poseen menor poder resolutivo genotípico que RAPDs, los valores reales de errores de clasificación podrían ser aún superiores.

Todo error de clasificación de material genético valioso, evidentemente representa un elevado costo financiero, debido a la pérdida de potencial genético y productividad para los programas de mejoramiento genético forestal. Además, ellos implican dificultades operacionales e impactos económicos negativos para productores y viveristas. El análisis molecular mediante RAPDs permitiría subsanar tales inconsistencias operacionales.

\section{CONCLUSIONES}

1-. El género Salix presenta una elevada variabilidad al nivel molecular.

2-. RAPDs permiten el eficaz análisis molecular de especies forestales, y presentan ventajas competitivas sobre otras tecnologías alternativas.

3-. Mediante RAPDs la identificación de cada una de las procedencias estudiadas se hizo posible, lo cual permitiría reducir la tasa de errores de identificación clonal entre ellas.

\section{AGRADECIMIENTOS}

Se agradece el financiamiento FONDEF (D9611074) y FDI (PF-05) que hicieron posible este estudio, así como el interés y visión de la ingeniero forestal Marta Ábalos (INFOR-Santiago) para desarrollar marcadores moleculares para procedencias Salix.

\section{BIBLIOGRAFÍA}

Bucci, G. y P. Menozzi. 1993. Segregation analysis of random amplified polymorphic DNA (RAPDs) markers in Picea abies Karst. Mol. Ecol. 2:227:232.

Campos de Q., H. 1995. Marcadores moleculares: Conceptos. AgroSur. 23(2):173-185.

Campos de Q., H. Y H. Salvo. 1995. Marcadores moleculares: aplicaciones al mejoramiento genético de plantas. AgroSur. 23(2):173-185.

Castiglione, S.; C. Wang; G. Damiani; C. Bandi; S. Bisoffi and F. Sala. 1993. RAPDs fingerprinting for identification and for taxonomic studies of elite poplar (Populus spp.) clones. Theor. Appl. Genet. 87:54-59.

Comisión Nacional del Álamo. 1997. Noticiero Octubre-Diciembre. p 3

Dos Santos, J; J. Nienhuis; P. Skroch; J. Tivang y M. Slocum. 1994. Comparison of RAPDs and RFLP genetic markers in determining genetic similarity among Brassica oleracea L. genotypes. Theor. Appl. Genet. 87:909-915. 
Furman, B.; D. Grattapaglia; W. Dvorak y D. Omalley. 1997. Analysis of genetic relationships of Central American and Mexican pines using RAPDs markers that distinguish species. Mol. Ecol. 6:321-331

Grattapaglia, D. and R. Sederoff. Genetic linkage maps of Eucalyptus grandis and Eucalyptus urophylla using a pseudo-testcross: mapping strategy and RAPDs markers. Genetics. 137:11211137. (1994).

Khasa, P. and B. Dancik.: Rapid identification of white-Engelmann spruce species by RAPDs markers. Theor. Appl. Genet. 92:46-52. (1996).

Liu, Z. y G. Furnier. 1993. Comparison of allozyme, RFLP and RAPDs markers for revealing genetic variation within and between trembling aspen and bigfooth aspen. Theor. Appl. Genet. 87:987-105.

Mosseler,A.; K. Egger y G. Hughes. 1992. Low levels of genetic diversity in red pine confirmed by random amplified polymorphic DNA. Can. J. Forest Res. 22:1332-1337.

Ojeda, V., F. Ortega y H. Campos de Q. 1998. Marcadores moleculares en trébol rosado (Trifolium pratense L.) I. RAPDs. In: IV Congreso Nacional de Biotecnología, Talca. Pp 87.

Scheepers, D.; M-C. Eloy and M. Briquet. 1997. Use of RAPDs patterns for clone verification and in studying provenance relationships in Norway spruce (Picea abies). Theor. Appl. Genet. 94:480-485.

Teasdale, R. 1996. Priorities for application of biotechnology to plantation improvement. In: Somatic Cell Genetics and Molecular Genetics of Trees. M. Ahuja; W. Boerjan and D. Neale (Eds.). p 69-80

Yu, Y-L y Lin, T-Y. 1997. Construction of phylogenetic tree for Nicotiana species based on RAPDs markers. J. Plant Res. 187-193.

Van Den Ven, W. y R. Mc Nicol. 1995. The use of RAPDs markers for the identification of Sitka spruce (Picea sitchensis) clones. Heredity. 75:126-132.

Wheler, N. y K. Jech. 1992. The use of electrophoretic markers in forest research. New Forests. 6:311-328.

Williams, J. G. K; A. Kubelik; K. Livak; A. Rafalski y S. Tingey. 1990. DNA polymorphisms amplified by arbitrary markers are useful as genetic markers. Nucl. A. Res. 18: 6531-6535. 